SHORT REPORT

\title{
Environmental tobacco smoke and prevalent coronary heart disease among never smokers in the Scottish MONICA surveys

\author{
R Chen, R Tavendale, H Tunstall-Pedoe
}

Occup Environ Med 2004;61:790-792. doi: 10.1136/oem.2003.009167

\begin{abstract}
A lthough many studies have shown that environmental tobacco smoke (ETS) or passive smoking increases the risk of coronary heart disease (CHD),$^{1-6}$ there is still scepticism. This may be due to the small magnitude of the effect (about $25-35 \%$ increase $^{56}$ ), inadequate adjustment for confounding variables, and misclassification of passive smoking. To estimate exposure, the majority of previous studies used only self report, without any biochemical markers, which could result in misclassification errors arising both from respondent and investigator biases. We analysed data from the Scottish MONICA surveys to see whether prevalent coronary heart disease is independently associated with passive smoking measured by self report, serum cotinine, and their combination.
\end{abstract}

\section{SUBJECTS AND METHODS}

Risk factor surveys were conducted for the Scottish MONICA Project in North Glasgow in 1986, 1989, 1992, and 1995. ${ }^{7}$ Subjects were randomly recruited from general practitioner lists after stratification into 10 year age and sex groups. A total of 1262 aged 25-64 participated in the first, 1397 in the second, 1958 (including 442 aged 65-74) in the third, and 1836 in the fourth, with response rates of $63.5 \%, 59.0 \%$, $65.5 \%$, and $65.7 \%$ respectively. Participants completed a personal health record that incorporated questions on sociodemographic and health status including details of active and passive smoking, the Rose chest pain questionnaire, and a food frequency questionnaire. ${ }^{38}$ Clinic examinations included electrocardiogram (ECG) and blood sampling for cotinine $^{7}$ and other biochemical analyses.

Altogether 1868 subjects claimed in the questionnaire to have never smoked. Fourteen with serum cotinine values $\geqslant 17.50 \mathrm{ng} / \mathrm{ml}$ were excluded as probable smokers. ${ }^{3}$ Subjects in surveys 1 and 2 were asked "Have you been exposed to tobacco smoke from someone else in the last three days?". The four possible answers were: yes, a lot; yes, some; yes, a little; or no, none at all. We categorised them in reverse order

\section{Main messages}

- There was a weaker relation of CHD to serum cotinine than to self reported ETS; but when cotinine was detected, a dose-response relation was observed.

- The combination of self reported ETS and serum cotinine showed a clearer gradient of prevalent CHD with exposure.

- The association of CHD with ETS was independent of other cardiovascular confounders.

- The increased risk of CHD for those in full time employment seemed to be mainly from ETS exposure at work. as groups I, II, III, and IV. In surveys 3 and 4, the question was "Are you regularly exposed to tobacco smoke from other people?"; separate exposures at work, at home, and in other places were recorded, and a numerical score ranging from 1 to 4, corresponding to the groups above, was assigned for each defined location. To estimate total exposure, the three numerical scores were added together for subjects in full time employment; ${ }^{8}$ for the remaining subjects, the scores from at home and in other places were summed and then multiplied by a factor of 1.5 to make the total exposure (or score) equivalent to those in full time employment. This process gives a range of scores from 3 to 12. In order to combine the data from these surveys with those from the earlier ones, scores of 3-4 were defined as I, scores $5-6$ as II, scores 7-8 as III, and scores 9-12 as IV. ${ }^{8}$ In surveys 3 and 4, an additional question was asked: "On average, for how many hours a day are you exposed to other people's tobacco smoke?".

Serum cotinine levels were categorised into four groups using cut points defined previously: ${ }^{3}$ no detectable cotinine, I; $>0-1.05 \mathrm{ng} / \mathrm{ml}$, II; $1.06-3.97 \mathrm{ng} / \mathrm{ml}$, III; and 3.98-17.49 ng/ $\mathrm{ml}$, IV. Because self report may involve misclassification, and serum cotinine level, an indicator of recent exposure over 1-2 preceding days, may be affected by time since the last passive smoking exposure (which raises the question of whether it is ideal to take cotinine concentration as a sole index of ETS exposure), we have recently suggested that a combination of self report and serum cotinine would be a better measurement of ETS in adults. ${ }^{7}$ To generate the combined self report and cotinine score, a numerical score ranging from 1 to 4 was assigned to each participant for each of the self report and cotinine groups into which they had been categorised, and the two scores were added together giving a possible range of $2-8$, which were further categorised in ascending order as groups I-VII.

The SAS (Windows version 8.2; SAS Institute Inc., Cary, NC) statistical package was used to analyse the data. Similar to the previous analysis, ${ }^{3}$ coronary heart disease was categorised as questionnaire angina, undiagnosed CHD (included questionnaire angina), diagnosed CHD, and all CHD. Difference in prevalence of CHD between ETS groups were examined by the $\chi^{2}$ test. The association of CHD to ETS was examined using a binary logistic regression model.

\section{RESULTS}

Table 1 shows the prevalence and odds ratios (OR) for CHD in relation to passive smoking. Questionnaire angina shows a

\section{Policy implications}

- The study further endorses current policies of strictly limiting smoke in public areas. 


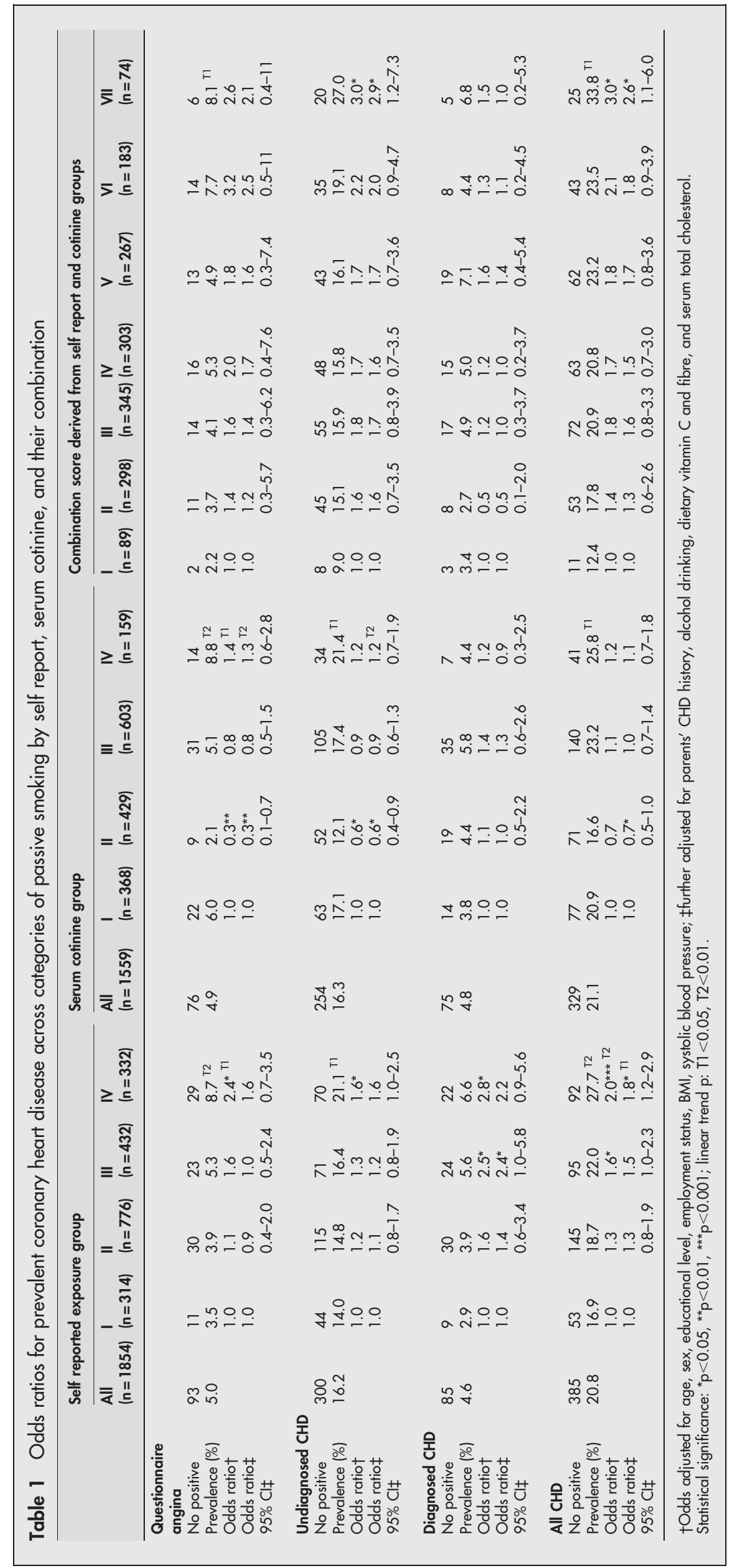


gradient of prevalence with increasing self reported exposure. The 300 cases of undiagnosed CHD further show a gradient of prevalence and a significant OR in the highest exposure group after adjustment for major coronary risk factors. With smaller numbers, diagnosed CHD shows raised ORs in the exposure groups. When all CHD categories are combined there is a regular, significant gradient. Results for serum cotinine do not corroborate self report completely. There was a higher prevalence of questionnaire angina, undiagnosed CHD, and all CHD in subjects with no detectable cotinine compared to those having cotinine levels $>0-1.05 \mathrm{ng} / \mathrm{ml}$, while the prevalence of diagnosed CHD was lowest in the group with no detectable cotinine. In the categories of questionnaire angina, undiagnosed CHD, and all CHD a significant, clear gradient of increased CHD risk with cotinine concentrations was found for those having detectable cotinine.

Using the combination score derived from self report and serum cotinine values, we observed a strong increase in CHD with increasing exposure for the questionnaire angina, undiagnosed CHD, and all CHD groups (table 1). This trend was not seen in the diagnosed CHD group, probably due to the smaller numbers of cases in each group (the increasing trend could be found if exposure groups I-II, III-IV, and VVII were combined).

In surveys 3 and 4 (1114 subjects), we found a significant association of prevalent CHD with self reported ETS duration; compared to non-exposure, the multiple adjusted OR for all CHD (253 cases) was $1.2(0.8-1.9)$ for daily exposure of $>0-2$ hours, $1.6(1.0-2.6)$ for $3-5$ hours, and $1.7(1.0-2.8)$ for over 6 hours (trend $\mathrm{p}=0.138$ ), while the combination with serum cotinine score further showed a similar gradient (data on request). Furthermore, the data of 502 subjects in full time employment indicated that the increased risk of all CHD (72 cases) seemed to be more closely related to ETS exposure at work (the multiple adjusted OR 1.7, 0.9-3.2 for those having any exposure compared to the no exposure group) than at home $(1.2,0.7-2.2)$ or in other places $(1.0,0.4-2.3)$.

\section{DISCUSSION}

The current study using the combined measure identified a consistent, dose-response relation between ETS and CHD. However, in separate analyses of ETS estimated by either self report or serum cotinine, there was a closer relation of CHD to self report, which was consistent with previous findings in the cross sectional data analysis for the Scottish Heart Health Study. ${ }^{3}$ An unexpected finding was the higher prevalence of CHD in the group with no detectable cotinine when compared to the group with the lowest level of detectable cotinine. A possible explanation is that the no detectable cotinine group contained a number of CHD patients who avoided ETS exposure. Other contributing factors could be: (1) the effect of delay in time between last ETS exposure and blood sampling; (2) cotinine data were missing for fewer $(9 \%$ $v 29 \%$ ) of the MONICA $3 \& 4$ subjects than the surveys 1 and $2,{ }^{7}$ where the finding was less apparent (see below); and (3) the capillary chromatographic method of cotinine analysis adopted for MONICA $3 \& 4$ was more sensitive (survey 1 used a packed column method but survey 2 used the same method as MONICA $3 \& 4$ ). It is arguable that the non-exposed group (that is, no detectable cotinine) should (or not) be included for the dose-response trend analysis, and that by excluding the never exposed groups, the linear trends of the doseexposure relations would be more convincing if they showed a significant trend of higher risk with higher degrees of exposure. ${ }^{9}$ Restricting the data to subjects with detectable cotinine, we found a gradient of CHD with cotinine concentration in both MONICA surveys 3 and 4 (the multiple adjusted ORs for all CHD in cotinine groups II to IV were 0.9 $(0.5-1.3), 1.4(0.9-2.1)$, and $1.4(0.7-2.6)$; trend $\mathrm{p}=0.089)$ and MONICA surveys 1 and 2 (ORs were $0.4(0.2-0.8), 0.5$ $(0.3-0.9)$, and $0.7(0.3-1.6)$; trend $p=0.026)$. The magnitude of the gradient was similar to (or even greater than) that by self report in groups II to IV (see table 1).

In this study, the main limitations are its cross sectional design and a relatively small number of subjects to identify the small magnitude of the effects of ETS on CHD, which led to insufficient statistical power for some increased ORs and trends. A large cohort study using both self report, serum cotinine, and their combination is needed to further examine the effects. However, the standardised and extensive database from the MONICA surveys allowed analysis and control for many confounding cardiovascular variables. Four ETS exposure degrees for both self report and serum cotinine and seven exposure groups for their combination gave us an opportunity of more accurately estimating the adverse association, and would be one of the reasons ${ }^{8}$ to explain that our data showed a greater effect of passive smoking than other studies ${ }^{56}$ which mainly used dichotomised ETS exposure estimation. The adverse association identified in self report, serum cotinine, and their combination was dose dependent, and not substantially changed by multiple adjustment for confounding variables. The findings have been further confirmed in a 14 year follow up of cardiovascular mortality in our Scottish Heart Health Study. ${ }^{10}$ For those in full time employment, the relation between ETS at work and CHD seemed to be greater compared to exposure at home and at other places. These findings, together with our previous investigations, ${ }^{3} 8$ strongly support current attempts to minimise passive exposure to tobacco smoke and limit smoking in public areas.

\section{Authors' affiliations}

R Chen, R Tavendale, $\mathbf{H}$ Tunstall-Pedoe, Cardiovascular Epidemiology Unit, Institute of Cardiovascular Research, University of Dundee, Ninewells Hospital and Medical School, Dundee DDI 9SY, UK

Funding: Chief Scientist Office, Scottish Executive Health Department and British Heart Foundation

Correspondence to: $\operatorname{Dr} \mathrm{R}$ Chen, Department of Epidemiology and Public Health, UCL, 1-19 Torrington Place, London WC1E 6BT, UK; ruoling.chen@westminster-pct.nhs.uk

Accepted 24 March 2004

\section{REFERENCES}

1 Hole DJ, Gillis CR, Chopra C, et al. Passive smoking and cardiorespiratory health in a general population in the west of Scotland. BMJ 1989;299:423-7.

2 La Vecchia C, D'Avanzo B, Franzosi MG, et al. Passive smoking and the risk of acute myocardial infarction. Lancet 1993;341:505-6.

3 Tunstall-Pedoe H, Brown CA, Woodward M, et al. Passive smoking by self reported and serum cotinine and the prevalence of respiratory and coronary heart disease in the Scottish heart health study. J Epidemiol Community Health 1995;49:139-43.

4 Steenland K, Thun M, Lally C, et al. Environmental tobacco smoke and coronary heart disease in the American Cancer Sciety CPS-II cohort. Circulation 1996;94:622-8.

5 Law MR, Morris JK, Wald NJ. Environmental tobacco smoke exposure and ischaemic heart disease: an evaluation of the evidence. BMJ 1997;315:973-80.

6 Jousilahti P, Patja K, Salomaa V. Environmental tobacco smoke and the risk of cardiovascular disease. Scand J Work Environ Health 2002;28(suppl 2):41-51.

7 Chen R, Tavendale R, Tunstall-Pedoe H. Measurement of passive smoking in adults: self-reported questionnaire or serum cotinine? J Cancer Epidemiol Prev 2002; 7:85-95.

8 Chen R, Tunstall-Pedoe H, Tavendale R. Environmental tobacco smoke and lung function in employees who never smoked: the Scottish MONICA study. Occup Environ Med 2001;58:563-8.

9 Bailar JC. Passive smoking, coronary heart disease, and meta-analysis. N Engl J Med 1999;340:358-9.

10 Chen R, Tavendale R, Tunstall-Pedoe H. Passive smoking measured by self report, serum cotinine and their combination and 14-year cardiovascular mortality among never smokers in the Scottish Heart Health Study [abstract]. Circulation 2003; 108:IV 751. 